Results We found significantly higher serum ADMA levels but not serum hs-CRP levels in NBF when compared to BF group ( $\mathrm{p}$ $<0.05$ ). According to BMI data starting from the age of 12 months more overweight/ obese children were found in NBF children when compared to BF. Serum ADMA was inversely associated with HDL-cholesterol levels and breastfeeding duration in studied children $(\mathrm{p}<0.05)$. Positive correlation was found between ADMA and body fat mass $(p<0.05)$.

Conclusion In NBF children increased circulating ADMA is observed, however further studies are needed to assess whether breastfeeding duration affects body fat and other measures of body composition at older ages.

\section{PO-0713 SYSTEMIC EFFECTS OF ANTICHOLINERGIC - SYMPATHOMIMETIC EYE DROPS DURING SCREENING FOR RETINOPATHY OF PREMATURITY}

${ }^{1} \mathrm{~N}$ Tekin, ${ }^{1} \mathrm{~S}$ Celiloglu, ${ }^{2} \mathrm{H}$ Gursoy, ${ }^{2} \mathrm{~N}$ Erol, ${ }^{1} \mathrm{MA}$ Aksit. ${ }^{1}$ Department of Pediatrics Division of Neonatology, Eskisehir Osmangazi University Medical Faculty, Eskisehir, Turkey; ${ }^{2}$ Department of Ophthalmology, Eskisehir Osmangazi University Medical Faculty, Eskisehir, Turkey

\subsection{6/archdischild-2014-307384.1349}

Background and aims Anti cholinergic and sympathomimetic eye drops are widely used to achieve mydriasis. Normally systemic effects of these eye drops are ignorable but adverse events in preterm infants are reported. In this study during routine screening for retinopathy of prematurity (ROP), preterm infants were searched for the systemic effects of eye drops.

Methods The standard protocol was to instil 3 drops per eye which is a mixture of short acting tropicamide $0.5 \%$ with long acting cyclopentolate $1 \%$ and phenylephrine $2.5 \%$ ophtalmic solution in equal volumes. Each drop instilled at a 15 min interval before examination. Body temperature, heart rate, respiration, blood pressure, $\mathrm{spO} 2$, presence of flashing were recorded three times; before the instillation of eye drops, just before the examination and after an hour. Parents were informed about the adverse side effects and presence of complaints were asked after $24 \mathrm{~h}$ with the telephone interview. Data were analysed by 2 twoway ANOVA and independent samples t-test.

Results Forty eight (27 male +21 female) infants with birth weight $1498 \pm 432(720-2500) \mathrm{g}$ and gestational age 31,7 \pm 3.3 $(25-37)$ weeks were examined at postmentruel age of $41.95 \pm$ 4.74(34-58) weeks. Body temperature rised subsequently with each eye drop $(p=0.023)$. The change in other physiologic parameters were not statistically significant. Apnea over10 seconds were developed in 9 infants. Within $24 \mathrm{~h}$ gastrointestinal symptoms developed in 8 , discomfort/sleeplessness in 22 , hyperemia/discharge from the eye in 20 infants.

Conclusion It was concluded that doctors must be aware of the the systemic effects of mydriatic eye drops used in screening examination for ROP and parents have to be informed about these effects.

\section{P0-0714 RESPIROMETRY OF PLATELETS SUGGESTS MITOCHONDRIAL DISORDER IN A NEWBORN INFANT WITH LETHAL HEPATOPATHY AND ENCEPHALOPATHY}

${ }^{1} \mathrm{~N}$ Tomasic, ${ }^{1} \mathrm{H}$ Kotarsky, ${ }^{1} \mathrm{E}$ Hansson, ${ }^{2} \mathrm{E}$ Elmer, ${ }^{1} \mathrm{~V}$ Fellman. ${ }^{1}$ Department of Pediatrics, Lund University, Lund, Sweden; '2Department of Clinical Neurophysiology, Lund University, Lund, Sweden
Background and aims Rapid diagnosis of mitochondrial disorder is difficult in newborn infants with metabolic crisis. We studied whether respiratory chain disorder can be assessed from circulating platelets.

Methods A full-term girl of consanguineous parents was born after uncomplicated pregnancy (2690 g, Apgar 10/10/10). On day one she was transferred to NICU with metabolic acidosis (pH 7.11, $\mathrm{pCO}_{2}$ 2.8, BE -24, lactate $19 \mathrm{mmol} / \mathrm{l}$ ). CSF/plasma lactate ratio (5.3/6.9) was increased. Cerebral MRI revealed diffuse changes in pyramidal tract and internal capsule. On day 4 she developed hepatic failure, conjugated hyperbilirubinemia and slight hyperammonemia. Urine metabolic analysis revealed increased 3-methylglutaconic acid (160 mmol/mol creatinine), and 4-hydroxyphenyllacetate suggestive of mitochondrial disorder. Respirometry (Oroboros Oxygraph, SUIT-protocol) was performed on blood cells. Isolated mitochondria from fibroblasts and liver were assessed with Blue Native-PAGE (BNGE) for respiratory chain complex assembly. Intensive care was withdrawn because of deterioration, and postmortem biopsies performed.

Results Respirometry on platelets showed a borderline oxygen consumption. Histology of muscle was normal, liver was cholestatic with iron accumulation. In fibroblasts, respiratory chain complex assembly was normal, but in liver levels of Complexes I, III and IV were decreased. Whole genome sequencing identified the candidate genes Sycp2, Clybl and Foxred1. The deficient complexes all possess mtDNA encoded subunits thus nuclear encoded translator mutation or other mtDNA related mutation might be causative.

Conclusions Respirometry from blood cells might suggest mitochondrial dysfunction that can be verified by structural analyses of respiratory chain complexes from the target organ. Causative mutation might be achieved with next generation sequencing.

\section{P0-0715 WITHDRAWN}

\section{PO-0716 CONTINUOUS VENOVENOUS HEMODIAFILTRATION EXPERIENCE OF FOUR NEWBORNS}

${ }^{1}$ A Tugcu, ${ }^{2}$ ASLI Kantar, ${ }^{1}$ A Abbasoglu, ${ }^{1}$ AYSE Ecevit, ${ }^{1}$ A Tarcan, ${ }^{3}$ ESRA Baskin. ${ }^{1}$ Pediatrics Neonatology, Baskent University, Ankara, Turkey; ${ }^{2}$ Pediatrics Nephrolohy, Baskent University, Ankara, Turkey; ${ }^{3}$ Pediatrics Nephrology, Baskent University, Ankara, Turkey

\subsection{6/archdischild-2014-307384.1351}

Aim Conventional methods are the first treatment modalities of renal failure or metabolic diseases in newborns. If these modalities fail to treat, we start to use peritoneal dialysis (PD). Continuous venovenous hemodiafiltration (vvHDF) is used when PD can not be performed. Our continuous vvHDF experience of 4 patients in neonatal intensive care unit, is presented.

Case1: A male term newborn infant, having mapple syrup urine disease with a high serum leucine value after PD could not be performed, vvHDF was successfully provided. He was discharged from our hospital on 34th postnatal day.

Case2: A preterm newborn, having polycystic renal disease and could not use under PD and vvHDF was started on 13th postnatal day. He died due to ventilator associated pneumonia on 135th postnatal day.

Case3: A term newborn, having "polycystic renal disease" and could not perform PD, was referred to our unit for continuous vvHDF administration on 3rd postnatal day. vvHDF application was continued until his 61 st postnatal day. 